\title{
The differential tonic effect in interbeat interval during the foreperiod of a reaction time task
}

\author{
SELINA REDMAN \\ University of Newcastle, Newcastle, N.S.W., Australia \\ and \\ JOHN DUTCH \\ Victoria University, Wellington, New Zealand
}

\begin{abstract}
Systematic individual differences in the direction of the tonic interbeat-interval response have been reported to occur when the foreperiod of a signaled reaction time task is extended. The experiment reported here explores the replicability and stability of the differential tonic effect. Forty subjects were presented with 20 reaction time trials with a 32 -sec foreperiod duration. Half of the subjects showed tonic acceleratory responses, and half, deceleratory responses. At retesting 6 weeks later, the subjects showed the same-direction tonic response as that in the first session, indicating the stability of the differential tonic effect.
\end{abstract}

A phasic change in heart rate, consisting of an initial acceleration followed by a deceleration, typically occurs during the foreperiod of a signaled reaction time task (e.g., Obrist, Webb, \& Sutterer, 1969). More recently, it has been reported that when the duration of the foreperiod is extended, the phasic response becomes superimposed on large magnitude tonic changes in heart rate (Dutch \& Redman, 1983). The emergence of such tonic responses within a common experimental paradigm is of some interest, given the comments of Obrist and his colleagues (e.g., Obrist, 1976; Obrist, Gaebelein, Teller, Langer, Grignolo, Light, \& McCubbin, 1978) indicating that tonic changes may be of more significance than phasic responses in understanding cardiovascular function and the development of such disease states as hypertension, because of the large magnitude and sympathetic determination of tonic responses.

Dutch and Redman (1983) also reported individual differences in the direction of the tonic response, with half of the subjects showing an acceleratory and half a deceleratory heart rate response. Although individual differences in cardiovascular responses have been previously studied during a reaction time task (e.g., Light \& Obrist, 1980), changes in response levels have typically been recorded across all phases of blocks of unsignaled reaction time tasks and investigations have concentrated primarily on differences between individuals only in terms of degree

This research was supported by Grant 200/13279 from the New Zealand Scientific Research Distribution Committee, Grant 78/263 from New Zealand University Grants Committee, and V.T.U internal research grants. S. Redman's mailing address is: Department of Behavioural Science in Relation to Medicine, University of Newcastle, Newcastle, N.S.W. 2308, Australia. of reactivity. Therefore, the demonstration of bidirectional tonic changes in heart rate specific to the foreperiod of a reaction time task are both novel and, given the magnitude of the effect, of potential importance.

The experiment reported here was designed to replicate the differential tonic effect observed by Dutch and Redman (1983), and to explore the stability across time of the individual differences in direction. Two additional procedural manipulations were used. First, during acquisition, we explored the extent to which the tonic response was dependent upon the later buttonpress response by introducing a procedure, similar to partial reinforcement (PRF), in which the imperative signal followed the countdown sequence on only a few randomly selected trials. Second, to reduce the effects of Phase 1 acquisition training on the later retest trials, a series of trials with the countdown sequence but no buttonpress were presented immediately after the acquisition phase.

\section{METHOD}

\section{Subjects}

Forty Stage 2 psychology students participated in this experiment in partial fulfillment of their course requirements.

\section{Equipment and Physiological Recording Procedures}

The presentation of stimuli and data collection were controlled by a Hewlett-Packard 9835B minicomputer. All stimuli were displayed via a Hewlett-Packard 9835A graphics terminal and screen.

The ECG signal was detected using Lead II with Beckman electrodes, and the resulting signal was filtered and amplified. Interbeat interval (IBI) was measured between successive $R$ waves of the ECG using a Hewlett-Packard counter-timer with millisecond accuracy.

\section{Procedure}

All subjects attended an initial 1-h session, during which Phases 1 and 2 of the experiment were run. Upon arrival at the laboratory, sub- 
jects were assigned randomly to either the partial or the continuous reinforcement group. The subjects were comfortably seated in a sound- and temperature-controlled room, the ECG electrodes were attached, and headphones, through which music was played for the duration of the experiment, were positioned. Instructions were displayed on the graphics terminal throughout this procedure and until data collection began. The instructions requested the subject to keep still and explained the task, stating that the subject was to press a reaction time button as fast as possible in response to the imperative signal.

During all phases of the experiment, each trial consisted of a 10-sec baseline period, a 32-sec CS period, and a 32-sec postresponse period. The CS consisted of the numerical sequence 4, 3,2,1 displayed consecutively on the screen of the graphics terminal. Each number was 4 cm high. At CS onset, a brief tone was sounded and " 4 " was displayed on the screen for $8 \mathrm{sec}$ and then replaced by a " 3 ." This process was repeated throughout the sequence, with each number being displayed for $8 \mathrm{sec}$. The imperative signal was the word "GO" displayed on the screen, in response to which the subjects were required to depress the reaction time button. Following each buttonpress, the speed of the reaction time was briefly displayed. Between trials, the screen was blank.

Phase 1: Acquisition. Phase 1 consisted of 20 acquisition trials, with each trial as described above. For the continuous reinforcement (CRF) group, the imperative signal followed every CS sequence, whereas for the partial reinforcement (PRF) group, the imperative signal was displayed on only $25 \%$ of the acquisition trials.

Phase 2: No response. Immediately following Phase 1, 20 trials were run, during which the subject was not required to press the RT button after the countdown sequence.

After the completion of Phases 1 and 2, the tonic change during the CS period was evaluated for each subject. Over the last 10 acquisition trials, the median change from baseline was calculated for each of the four consecutive 8-sec periods. Each subject was then classified as an accelerator or a decelerator on the basis of two criteria: (1) whether the majority of their four 8-sec medians were above or below baseline, and (2) if there was no clear majority, whether the mean of the last two medians was above or below baseline.

Phase 3: Retest. Six weeks after completion of Phase 1 and Phase 2, the 20 subjects exhibiting the largest tonic responses in Phase 1 were required to again attend the laboratory. Large-magnitude tonic responders were selected on the basis of the magnitude of the change from baseline during the last 8-sec of the $\mathrm{CS}$ period in the second 10 acquisition trials in Phase 1 . The 20 subjects were selected so that five were CRF accelerators, five CRF decelerators, five PRF accelerators, and five PRF decelerators.

Attachment of electrodes and presentation of instructions were performed in a manner identical to that prior to Phases 1 and 2. Each subject was then presented with 10 acquisition trials identical to those they had received in Session 1.

\section{RESULTS AND DISCUSSION}

During Phase 1, a consistent tonic IBI response was evident for 36 of the 40 subjects. It was therefore necessary to use classification criterion 2 to categorize only 4 subjects, and the following analysis is based on all 40 subjects. The classification procedure resulted in the designation of $10 \mathrm{CRF}$ and 10 PRF subjects as accelerators and $10 \mathrm{CRF}$ and $10 \mathrm{PRF}$ subjects as decelerators. There were no differences in mean baseline IBI between accelerators and decelerators.

For each subject, IBI during the last 10 acquisition trials was converted to change scores by subtraction from his or her own baseline IBI over the same 10 trials. The following analysis therefore represents the mean deviation from each subject's own baseline during the countdown period. Figure 1 shows the mean change from

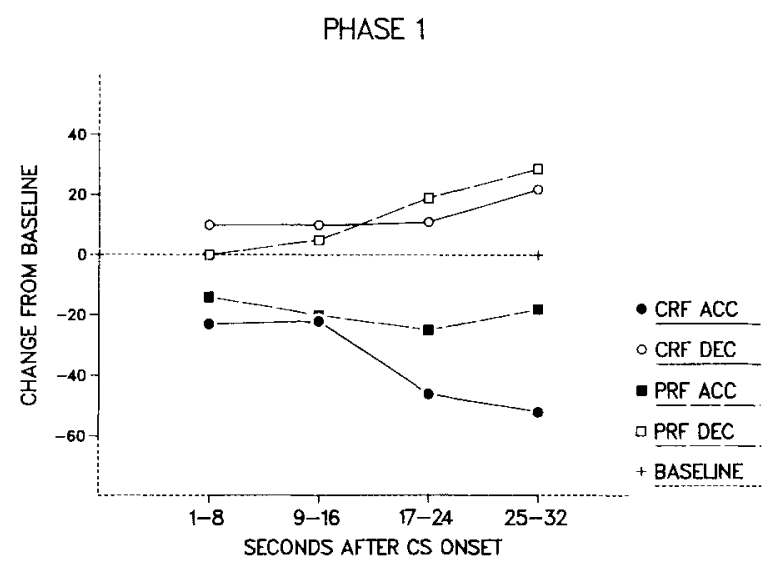

Figure 1. Mean change from baseline in milliseconds during consecutive 8 sec blocks after CS onset over all subjects in Phase 1.

baseline during the last 10 acquisition trials separately for CRF and PRF subjects and for accelerators and decelerators.

It is evident from Figure 1 that both the acceleratory and deceleratory tonic response can be observed during the first $8 \mathrm{sec}$ of the countdown period and continues to increase in magnitude throughout the entire $32 \mathrm{sec}$.

The data from Phase 1 thus replicate the findings of Dutch and Redman (1983), demonstrating tonic changes in IBI when the duration of the foreperiod of a signaled reaction time task was extended. Again, individual differences in the direction of the tonic response were observed during acquisition, with half of the subjects showing an acceleratory and half a deceleratory response.

To compare the effects of the PRF and CRF conditions on the acceleratory and deceleratory tonic responses, the change from baseline during the last $8 \mathrm{sec}$ of the foreperiod were evaluated using a two-tailed t test. Neither the accelerators $[t(18)=1.99, n$.s.] nor the decelerators $[t(18)=0.83$, n.s. $]$ showed any difference in the magnitude of the response in the PRF as opposed to the CRF condition. Thus, the differential tonic effect does not appear to depend simply upon the following buttonpress requirement.

During Phase 3, the retest, the median change from baseline over the 10 retest trials was calculated for each subject, as described for Phase 1. Figure 2 shows the change from baseline of the maximum accelerators and decelerators during Phases 1 and 3 .

When the response during the last $8 \mathrm{sec}$ was compared with baseline using a repeated measures $t$ test (one-tailed), a highly significant tonic decrease in IBI was observed for the accelerators $[t(9)=3.87, p<.005]$ and an increase in IBI was found for the decelerators $[t(9)=2.39$, $\mathrm{p}<.025]$. Two distinct groups were thus still evident, with accelerators showing a significantly greater decrease in IBI than decelerators [ $t(18)=4.34, \mathrm{p}<.005]$. The Phase 3 retest data thus indicate that the individual differences in direction of the tonic response are stable across time. 
PHASE 1

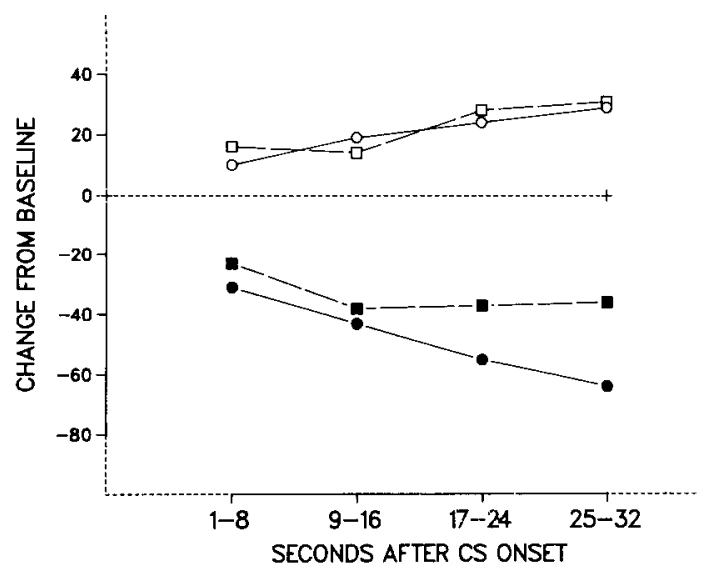

PHASE 3

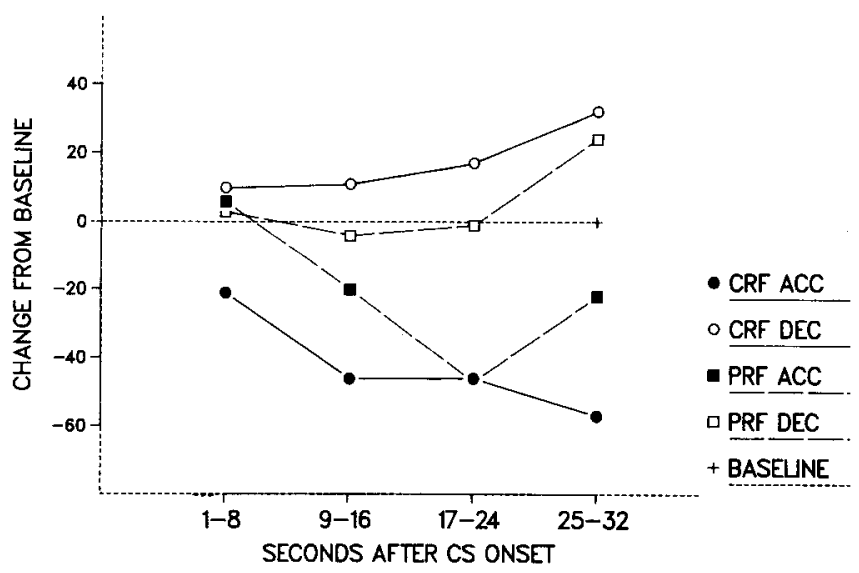

Figure 2. Mean change from baseline in milliseconds during consecutive 8-sec blocks after CS onset for large-magnitude accelerators and decelerators during Phases 1 and 3.

The differential tonic effect reported by Dutch and Redman (1983) therefore appears to be both replicable and stable. Further investigations should aim to determine the cause and significance of this effect.

\section{REFERENCES}

Dutch, J., \& Redman, S. (1983). Tonic and phasic changes in interbeat interval and ECG transit time during the foreperiod of a reaction time task. Physiological Psychology, 11, 130-134.

Light, K. C., \& OBRIST, P. A. (1980). Cardiovascular response to stress: Effects of opportunity to avoid, shock experience and feedback. Psychophysiology, 17, 243-252.
OвRIST, P. A. (1976). The cardiovascular-behavioral interaction as it appears today. Psychophysiology, 13, 95-107.

Obrist, P. A., Gaebelein, C. J., Teller, E. S., Langer, A. W., Grignolo, A., Light, K. C., \& McCubBin, J. A. (1978). The relationship among heart-rate carotid $\mathrm{dP} / \mathrm{dt}$ and blood pressure in humans as a function of stress. Psychophysiology, 15, 102-115.

Obrist, P. A., Webb, R. A., \& SutTerer, J. (1969). Heart rate and somatic changes during oversive conditioning and a simple reaction time task. Psychophysiology, 5, 696-723.

(Manuscript received October 11, 1983; revision accepted for publication August 31, 1984.) 